\title{
Comparação entre dados de precipitação obtidos por satélites e por pluviômetros no Vale do Paraíba
}

\author{
Patrícia Mayumi Teramoto ${ }^{1}$ \\ Gilberto Fisch ${ }^{2}$ \\ Silvio Jorge Coelho Simões ${ }^{1}$ \\ ${ }^{1}$ Universidade Estadual Paulista "Júlio de Mesquita Filho" - UNESP/FEG \\ Av. Dr. Ariberto Pereira da Cunha, 333 - CEP 12516-410 - Guaratinguetá, SP, Brasil \\ \{civ06199, simoes\}@ feg.unesp.br \\ ${ }^{2}$ Instituto de Aeronáutica e Espaço (IAE) \\ Praça Marechal Eduardo Gomes, 50 - CEP 12228-904 - São José dos Campos, SP, Brasil \\ gfisch@iae.cta.br
}

\begin{abstract}
In the meteorological community, there are for free groups of data of global precipitation that could be used in hydrological cycle studies. These data show a good time and spacial resolution, furthermore they don't be fail. However, they need to be calibrated and validated against local observation. This work intended to develop a study that has used the gauge observation has been doing in the meteorological point in the UNITAU (Taubaté University) of 1983 until 2008 and compare them with GPCP (Global Precipitation Climatology Project) data base, monthly estimate for a period of 1979 until 2008, for trend analysis in the local due to changes in global climatology. The analysis have realized with traditional statistics. The vaildation of these data let to extend the actual series, fail and limited, for long periods. The correlation among the observation and estimate in the time's scale monthly and season that shows the best result were May $(\mathrm{R}=0,8890)$ for month and winter $(R=0,7725)$, respectively. The time series shows the maintenance of the hydrological regime.
\end{abstract}

Palavras-chave: global precipitation, GPCP data base.

\section{Introdução}

O Vale do Paraíba compreende cidades localizadas no estado de São Paulo e Rio de Janeiro, em sua porção paulista abriga um dos maiores pólos industriais, além de acolher áreas de pecuária extensiva, sendo sinônimo de desenvolvimento.

A região Sudeste está localizada na zona tropical do planeta Terra e caracteriza-se por múltiplas condições climáticas ao longo de todo o ano, apresentando variabilidade sazonal, característica do clima tropical úmido-seco ou tropical do Brasil central. No Vale do Paraíba, predomina o clima tropical do Brasil central sem seca, ou seja, há ocorrência de chuvas em todos os meses, mas com maior concentração no verão, período em que ocorrem temperaturas elevadas, provocando chuvas de origem convectiva. Por outro lado, no inverno ocorre uma redução da precipitação, sendo que as chuvas são de origem frontal.

Atualmente, a NASA (National Aeronautics and Space Administration), juntamente com outros órgãos internacionais de pesquisas meteorológicas, desenvolvem programas espaciais e satélites, os quais buscam gerar dados que possam trazer informações concretas sobre as mudanças climáticas que a Terra vem sofrendo e, dessa forma, determinar se este é um processo natural e evolutivo do planeta ou uma consequiência da ação humana.

Dentre estes programas cita-se o GPCP (Global Precipitation Climatology Project), o qual disponibiliza informações mensais de precipitação desde 1979, combinando dados in situ de precipitação obtidas por pluviômetros e estimativas de precipitação através de imagens de 
satélites gerando estimativas globais com uma resolução espacial de 2,5 x 2,5 graus, o que representa $250 \mathrm{~km}$ x $250 \mathrm{~km}$ (Adler et al. 2003). A grande vantagem destas estimativas é que são aproveitados os aspectos bons que cada fonte de dados oferece após a correta manipulação.

O conhecimento do regime pluviométrico da região, através de dados históricos observacionais e estimado, permite identificar possíveis tendências locais de precipitação como descrito por Gruber e Levizzani (2006).

Os dados de precipitação pluviométrica no posto de coleta da UNITAU - Universidade de Taubaté são registrados desde 1983, o que nos permite adotar neste trabalho uma série histórica de somente 26 anos (1983-2008). Tais dados apresentam falhas devido a problemas instrumentais e operacionais e, também são realizados pontualmente, o que não é apropriado para chuvas convectivas podendo ocasionar erros (FISCH et al. 2006).

O objetivo principal deste trabalho é comparar dados de precipitação observacionais e estimados para o Vale do Paraíba obtidos respectivamente por pluviômetro e por satélites a fim de identificar possíveis tendências locais.

\section{Metodologia}

2.1 Coleta e análise de dados

Os dados disponibilizados pelo GPCP foram obtidos na página http://disc2.nascom.nasa.gov/Giovanni/tovas/rain.GPCP.2.shtml, para o quadrilátero compreendido entre as latitudes $22,5^{\circ}$ e $25^{\circ} \mathrm{S}$ e as longitudes $45^{\circ}$ e $47,5^{\circ} \mathrm{W}$ e podem ser vistos na Tabela 1. Estes se referem a uma área definida por coordenadas geográficas prédeterminadas pela resolução espacial do satélite e por produtos de origem de previsão numérica de tempo. Por outro lado, os dados medidos in situ na UNITAU foram fornecidos pelo Departamento de Ciências Agrárias daquela Universidade, são medidas pontuais realizadas com um pluviômetro convencional (Ville de Paris) e são apresentados na Tabela 2. Folhes e Fisch (2006) fazem uma análise detalhada destes dados observacionais, inclusive com estudos sobre a tendência temporal do regime pluviométrico.

A série apresentada pelos dados observacionais limita-se a 1983 a 2008, mas não apresenta falhas de coleta de dados, sendo utilizada para a calibração e validação dos dados estimados. A série completa de 1979 a 2008 do GPCP será utilizada para a análise de tendências de precipitação.

Tabela 1 - Totais mensais (mm) de precipitação obtidos pelo GPCP.

\begin{tabular}{|c|c|c|c|c|c|c|c|c|c|c|c|c|}
\hline Ano & JAN & FEV & MAR & ABR & MAI & JUN & JUL & AGO & SET & OUT & NOV & DEZ \\
\hline 1979 & 156,1 & 221,6 & 126,8 & 85,6 & 116,0 & 23,6 & 90,8 & 94,1 & 132,3 & 126,8 & 184,9 & 196,0 \\
\hline 1980 & 221,9 & 233,7 & 118,1 & 124,3 & 31,9 & 71,0 & 58,1 & 91,6 & 96,8 & 113,5 & 139,0 & 272,2 \\
\hline 1981 & 222,4 & 112,6 & 207,8 & 91,7 & 29,0 & 68,2 & 47,9 & 41,9 & 31,0 & 187,2 & 214,3 & 208,7 \\
\hline 1982 & 230,1 & 205,3 & 253,2 & 87,4 & 64,4 & 68,0 & 54,1 & 52,3 & 28,7 & 166,0 & 168,6 & 293,0 \\
\hline 1983 & 228,2 & 232,3 & 274,5 & 156,8 & 186,5 & 183,1 & 82,9 & 21,6 & 248,1 & 154,9 & 142,5 & 260,4 \\
\hline 1984 & 182,5 & 40,5 & 98,5 & 125,1 & 67,5 & 22,0 & 43,2 & 100,5 & 104,9 & 60,0 & 124,1 & 195,5 \\
\hline 1985 & 270,3 & 244,7 & 248,2 & 89,2 & 58,7 & 32,0 & 14,6 & 22,6 & 87,6 & 60,2 & 138,8 & 162,9 \\
\hline 1986 & 163,9 & 260,4 & 257,8 & 91,7 & 103,5 & 22,0 & 80,8 & 88,7 & 63,8 & 62,1 & 152,3 & 346,6 \\
\hline 1987 & 271,9 & 176,7 & 139,9 & 173,9 & 196,2 & 124,0 & 30,4 & 17,6 & 104,6 & 112,9 & 80,4 & 170,9 \\
\hline 1988 & 245,8 & 238,3 & 133,4 & 159,5 & 143,7 & 71,7 & 20,3 & 5,0 & 51,9 & 153,9 & 89,7 & 173,1 \\
\hline 1989 & 229,7 & 240,0 & 177,1 & 118,5 & 89,1 & 62,4 & 114,6 & 26,1 & 119,9 & 62,9 & 114,0 & 139,4 \\
\hline
\end{tabular}




\begin{tabular}{|c|c|c|c|c|c|c|c|c|c|c|c|c|}
1990 & 225,8 & 125,2 & 179,0 & 90,1 & 65,3 & 47,3 & 106,9 & 55,3 & 92,5 & 100,3 & 90,7 & 107,6 \\
\hline 1991 & 237,8 & 204,8 & 326,4 & 94,2 & 50,4 & 58,0 & 36,7 & 31,2 & 79,9 & 146,5 & 71,4 & 207,6 \\
\hline 1992 & 163,0 & 104,0 & 202,3 & 68,8 & 105,5 & 9,7 & 67,1 & 38,5 & 125,2 & 180,6 & 198,4 & 140,0 \\
\hline 1993 & 208,8 & 269,9 & 170,6 & 95,4 & 71,9 & 78,3 & 20,8 & 36,3 & 186,7 & 109,1 & 104,0 & 175,6 \\
\hline 1994 & 225,7 & 187,6 & 227,3 & 113,1 & 82,4 & 58,0 & 37,8 & 15,2 & 31,7 & 96,3 & 147,2 & 236,3 \\
\hline 1995 & 315,4 & 367,1 & 241,1 & 74,2 & 91,2 & 54,8 & 46,8 & 58,5 & 78,8 & 189,4 & 126,0 & 243,6 \\
\hline 1996 & 243,4 & 259,7 & 346,0 & 99,1 & 62,1 & 58,9 & 24,0 & 42,8 & 167,2 & 157,0 & 147,9 & 257,7 \\
\hline 1997 & 304,5 & 93,1 & 78,6 & 57,0 & 79,1 & 101,0 & 15,1 & 48,0 & 120,3 & 91,5 & 165,0 & 188,6 \\
\hline 1998 & 185,8 & 305,9 & 180,9 & 69,1 & 104,2 & 31,8 & 18,8 & 62,1 & 108,7 & 218,3 & 59,2 & 192,2 \\
\hline 1999 & 326,1 & 260,4 & 174,5 & 80,9 & 50,2 & 67,6 & 23,7 & 16,0 & 101,8 & 54,8 & 100,9 & 171,9 \\
\hline 2000 & 304,2 & 223,2 & 157,3 & 17,7 & 21,8 & 16,0 & 41,2 & 81,8 & 140,5 & 69,9 & 185,0 & 251,5 \\
\hline 2001 & 203,3 & 209,2 & 158,2 & 34,9 & 111,3 & 37,7 & 41,3 & 46,6 & 62,1 & 175,4 & 165,2 & 203,6 \\
\hline 2002 & 264,5 & 214,6 & 164,6 & 62,5 & 76,1 & 57,9 & 37,9 & 90,8 & 79,0 & 109,5 & 212,2 & 222,4 \\
\hline 2003 & 370,3 & 128,2 & 154,8 & 75,9 & 48,5 & 15,6 & 43,3 & 42,6 & 28,8 & 122,5 & 162,3 & 188,1 \\
\hline 2004 & 240,2 & 273,3 & 155,8 & 147,7 & 87,0 & 50,1 & 88,3 & 7,6 & 34,4 & 109,2 & 156,6 & 230,5 \\
\hline 2005 & 314,4 & 130,6 & 193,4 & 139,8 & 136,0 & 47,9 & 52,1 & 24,8 & 147,8 & 145,9 & 106,4 & 175,8 \\
\hline 2006 & 250,9 & 233,5 & 234,2 & 50,2 & 28,3 & 21,3 & 57,0 & 24,9 & 92,8 & 120,7 & 226,0 & 223,8 \\
\hline 2007 & 277,4 & 194,1 & 134,0 & 105,5 & 75,9 & 20,5 & 136,5 & 21,9 & 16,4 & 106,6 & 194,3 & 200,8 \\
\hline 2008 & 342,4 & 267,0 & 199,2 & 149,0 & $*$ & $*$ & $*$ & $*$ & $*$ & $*$ & $*$ & $*$ \\
\hline
\end{tabular}

Tabela 2 - Totais mensais (mm) de precipitação obtidos na UNITAU.

\begin{tabular}{|c|c|c|c|c|c|c|c|c|c|c|c|c|}
\hline Ano & JAN & FEV & MAR & ABR & MAI & JUN & JUL & AGO & SET & OUT & NOV & DEZ \\
\hline 1983 & 258 & 193 & 136,5 & 111,5 & 127,5 & 151 & 53 & 11,5 & 266 & 51,5 & 109,3 & 157,5 \\
\hline 1984 & 103,5 & 63 & 67 & 109 & 90,5 & 0 & 3,5 & 48,5 & 84,5 & 24,5 & 98,5 & 161 \\
\hline 1985 & 275 & 200 & 189,5 & 44 & 57 & 10,5 & 2,5 & 9 & 75,5 & 117 & 109,5 & 237 \\
\hline 1986 & 142,5 & 134 & 268 & 83,5 & 58 & 7 & 26,5 & 81,5 & 39,5 & 42,5 & 115,5 & 249,5 \\
\hline 1987 & 293,5 & 215 & 111 & 221 & 181 & 129 & 11,5 & 1,5 & 50 & 103,5 & 85,5 & 70,5 \\
\hline 1988 & 196 & 293 & 213 & 97 & 152,5 & 31 & 0 & 0 & 34 & 153,5 & 71,5 & 178 \\
\hline 1989 & 231,5 & 340,3 & 247,3 & 113,3 & 72,9 & 85,5 & 84,2 & 79,4 & 83,7 & 19,2 & 135,8 & 193,8 \\
\hline 1990 & 161,7 & 123,2 & 132,8 & 74,5 & 21,3 & 5 & 67,8 & 76,2 & 117,6 & 83,3 & 107,5 & 95,3 \\
\hline 1991 & 301,9 & 162,4 & 356,3 & 84,8 & 20 & 32,9 & 9,8 & 14,1 & 118 & 141,3 & 96,8 & 178,1 \\
\hline 1992 & 166,5 & 129,8 & 159,8 & 80 & 53,7 & 0 & 75,2 & 23,7 & 125,6 & 114,5 & 210,5 & 130,2 \\
\hline 1993 & 245,6 & 250,6 & 300,2 & 131,7 & 49 & 31,9 & 12,3 & 14,7 & 145,5 & 84,5 & 79,9 & 86,1 \\
\hline 1994 & 216,7 & 72,4 & 151,3 & 100,1 & 89,2 & 40,5 & 21,7 & 0 & 1,1 & 96,7 & 132,4 & 298,2 \\
\hline 1995 & 151 & 353,5 & 197,8 & 31 & 67,5 & 8,1 & 63,9 & 11,9 & 38,2 & 266,5 & 120,3 & 183,1 \\
\hline 1996 & 215,8 & 229,3 & 364,8 & 48,1 & 31,9 & 22,9 & 5,1 & 26,1 & 149,2 & 124,1 & 189,5 & 201,3 \\
\hline 1997 & 223,5 & 49,2 & 70,4 & 25,8 & 57,4 & 69,3 & 9 & 18,4 & 112,6 & 63,8 & 243,8 & 142,4 \\
\hline 1998 & 120,7 & 254,3 & 158,4 & 45,6 & 89,1 & 12,4 & 12,3 & 10,6 & 141,1 & 211,1 & 66,4 & 130,5 \\
\hline 1999 & 377,6 & 401,4 & 80,5 & 40,6 & 19 & 68,6 & 11 & 4,8 & 65 & 26,5 & 71,4 & 150 \\
\hline 2000 & 342 & 169,1 & 260,1 & 8,3 & 9,4 & 0,4 & 60,5 & 92,4 & 90,4 & 56 & 227,8 & 235,9 \\
\hline 2001 & 109,9 & 108,6 & 123,4 & 25,8 & 81 & 6,2 & 31 & 60,7 & 71,1 & 137,1 & 121,1 & 270,4 \\
\hline 2002 & 265,3 & 192,7 & 113,1 & 56,8 & 71,9 & 1,8 & 9,2 & 78,8 & 60,2 & 99,6 & 301 & 114,9 \\
\hline
\end{tabular}




\begin{tabular}{|c|c|c|c|c|c|c|c|c|c|c|c|c|}
\hline 2003 & 288,2 & 48,5 & 131,1 & 23,7 & 29,5 & 1,3 & 15,7 & 25,7 & 22,9 & 188,4 & 170,3 & 88,1 \\
\hline 2004 & 251,1 & 276,4 & 172,7 & 137,6 & 87,9 & 52,8 & 84,6 & 3,7 & 41,9 & 152,9 & 225,9 & 126,7 \\
\hline 2005 & 331,8 & 78,5 & 121,6 & 72,6 & 105,2 & 18,1 & 27,7 & 1,7 & 94,5 & 131,1 & 139,4 & 198 \\
\hline 2006 & 177,4 & 296,5 & 206,8 & 78,6 & 34,2 & 5,8 & 57,9 & 23,1 & 73,6 & 128,7 & 218,6 & 177,2 \\
\hline 2007 & 150,9 & 94,5 & 85,1 & 137,4 & 55,2 & 19,5 & 128,2 & 1,2 & 24,1 & 140,8 & 263,9 & 110,2 \\
\hline 2008 & 230,4 & 189,1 & 266,7 & 173,6 & 52,4 & 31,8 & 0 & 91,9 & 59,6 & 93,1 & 246,6 & 313,5 \\
\hline
\end{tabular}

A partir dos dados coletados foram confeccionados gráficos que correlacionam os dados observacionais com as estimativas e com o cálculo de dois parâmetros, sendo Desvio1 o viés estatístico da amostra e o Desvio 2 este mesmo viés normalizado. Estes desvios, apresentados nas equações 1 e 2, foram calculados com o auxílio do software Excel para as escalas de tempo mensal, sazonal e anual. O termo observação corresponde aos dados pluviométricos medidos na UNITAU e a estimativa aos dados do GPCP.

Desvio $1=$ Estimativa - observação

Desvio $2=\frac{\text { Estimatiza-Observą̧äo }}{\text { Obsarvag̨ăo }}$

\section{Resultados}

Como se pode perceber no gráfico de precipitação média sazonal (Figura 2) há variação considerável entre as estações do ano como já preconizado na literatura. Tal ocorrência também pode ser observada no gráfico de precipitação média mensal (Figura 1). Para o ano de 2008 ainda não está disponível a série completa de dados; por isso, esta série não foi considerado na análise anual e sazonais correspondentes ao outono, inverno e primavera. A Figura 3 apresenta a precipitação total anual.

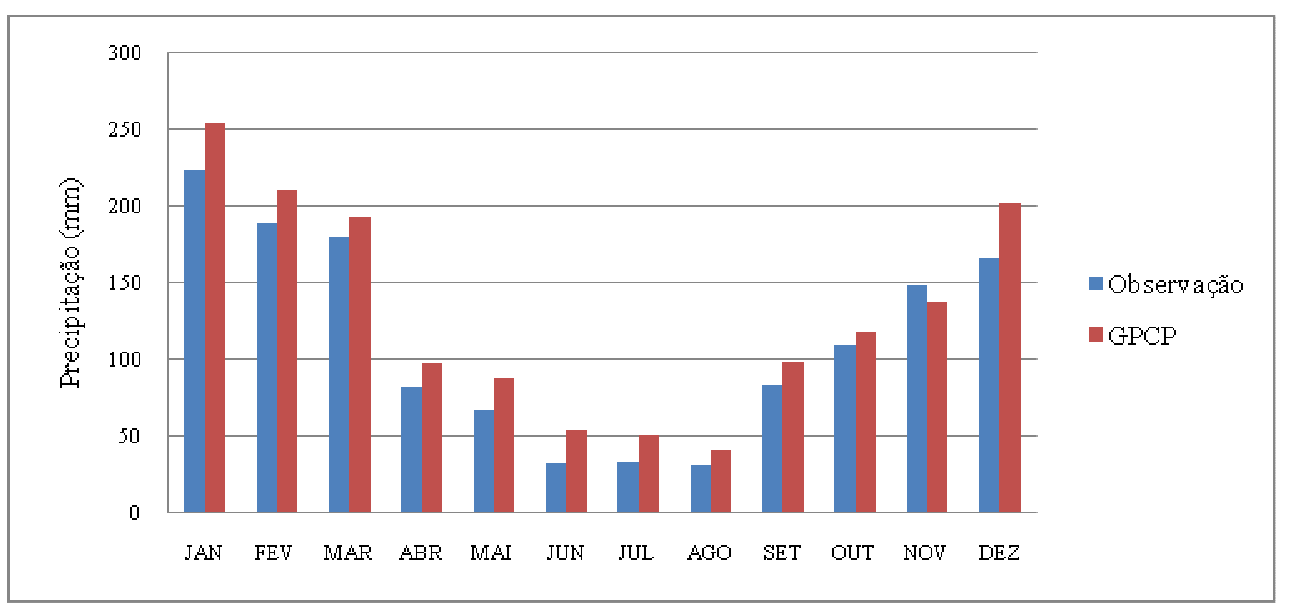

Figura 1 - Precipitação média mensal. 


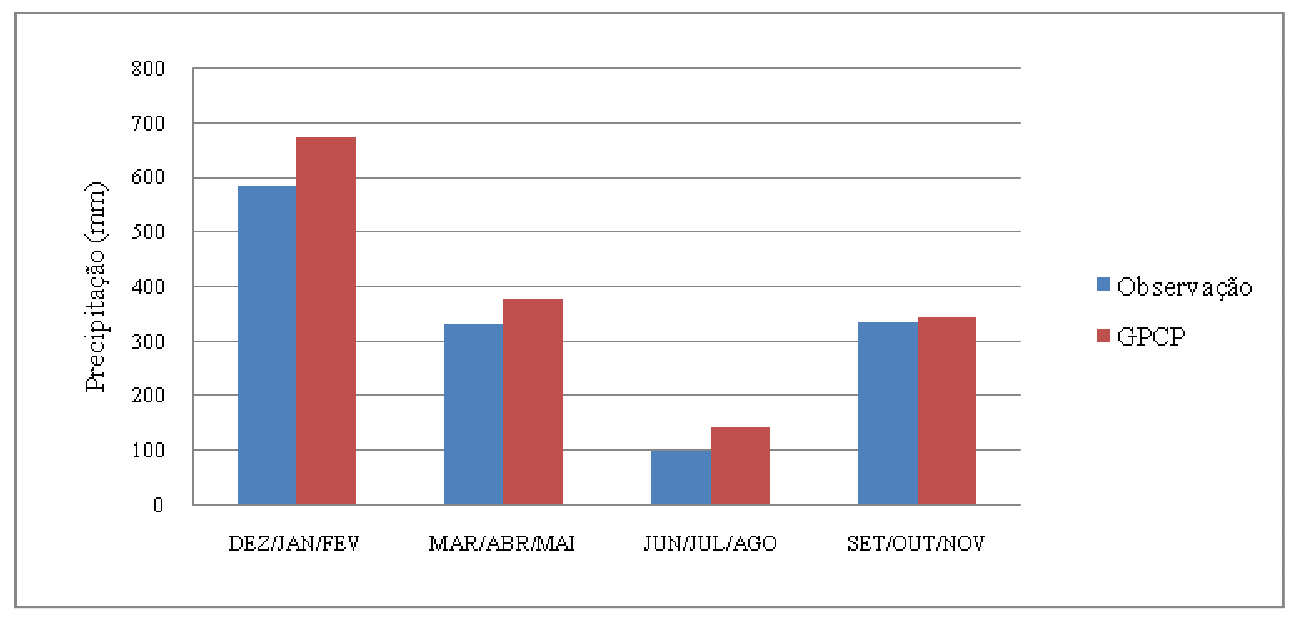

Figura 2 - Precipitação média sazonal.

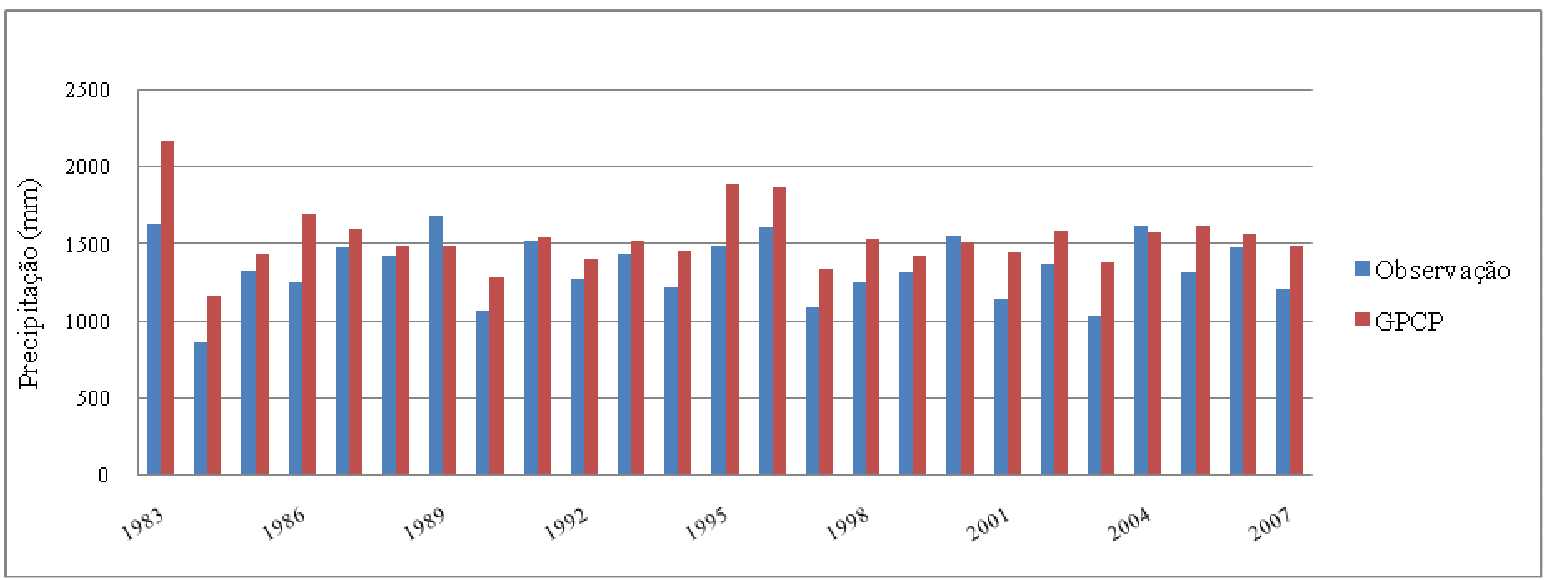

Figura 3 - Série temporal da Precipitação no Vale do Paraíba.

A Figura 4 mostra a precipitação estimada pelo GPCP desde 1979 e indica a manutenção do regime pluviométrico, segundo o software KyPlot versão 2.0, disponibilizado ao público gratuitamente, através da utilização do Método de Mann-Kendal. A tendência de precipitação ao longo dos últimos 29 anos apresentou $\tau=-0,0394$.

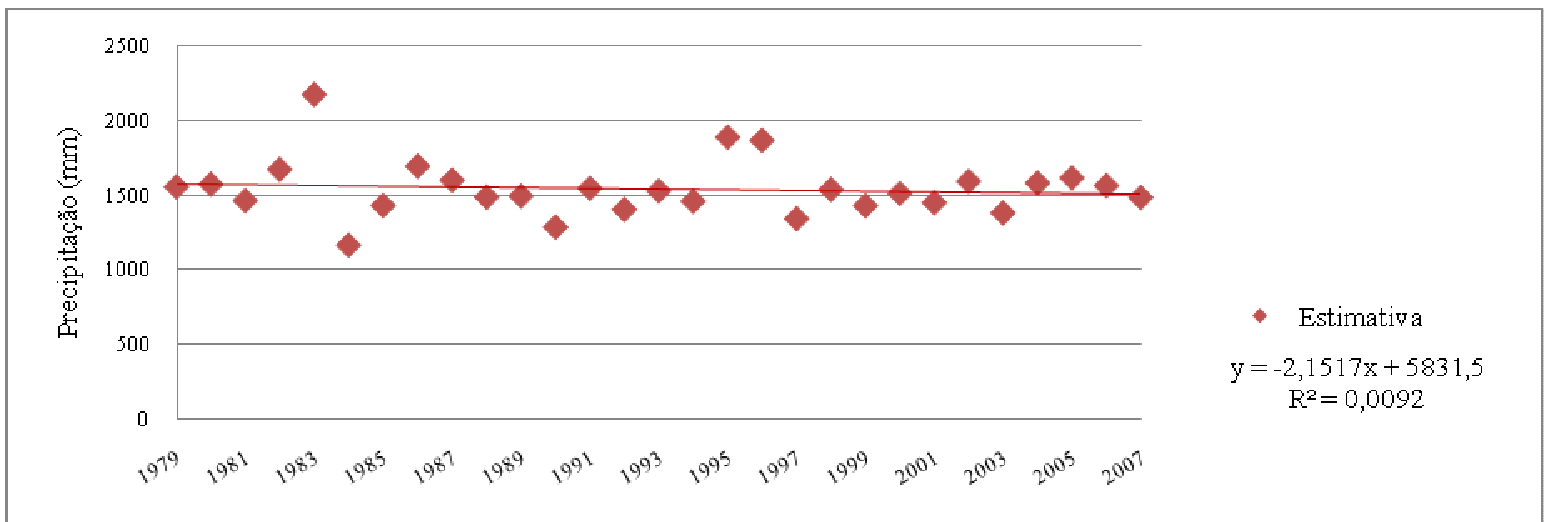

Figura 4 - Série temporal completa dos dados de precipitação anual.

Nas Tabelas 3, 4 e 5, são apresentadas as correlações obtidas entre os dados observacionais e os estimados, para as escalas de tempo mensal, sazonal e anual, respectivamente. 
Tabela 3 - Resultados de coeficiente de correlação (escala mensal).

\begin{tabular}{|c|c|c|c|}
\hline Mês & $\mathrm{R}$ (Observação x Estimativa) & $\mathrm{R}$ (Observação $\mathrm{x}$ Desvio 1) & $\mathrm{R}$ (Observação x Desvio 2) \\
\hline Janeiro & 0,6005 & $-0,6860$ & $-0,7480$ \\
\hline Fevereiro & 0,7508 & $-0,6773$ & $-0,6717$ \\
\hline Março & 0,6674 & $-0,6632$ & $-0,6947$ \\
\hline Abril & 0,7850 & $-0,6076$ & $-0,7059$ \\
\hline Maio & 0,8890 & $-0,1998$ & $-0,6251$ \\
\hline Junho & 0,8886 & $-0,3325$ & $-0,4018$ \\
\hline Julho & 0,8667 & $-0,2992$ & $-0,5143$ \\
\hline Agosto & 0,7083 & $-0,5262$ & $-0,5243$ \\
\hline Setembro & 0,8834 & $-0,3400$ & $-0,3380$ \\
\hline Outubro & 0,7367 & $-0,6523$ & $-0,7025$ \\
\hline Novembro & 0,8400 & $-0,7760$ & $-0,6309$ \\
\hline Dezembro & 0,4429 & $-0,6583$ & $-0,7575$ \\
\hline
\end{tabular}

Tabela 4 - Resultados de coeficiente de correlação (escala sazonal).

\begin{tabular}{|c|c|c|c|}
\hline Estação & $\mathrm{R}$ (Observação x Estimativa) & $\mathrm{R}$ (Observação x Desvio 1) & $\mathrm{R}$ (Observação x Desvio 2) \\
\hline Verão & 0,6141 & $-0,6824$ & $-0,7429$ \\
\hline Outono & 0,6693 & $-0,4761$ & $-0,6394$ \\
\hline Inverno & 0,7725 & $-0,5746$ & $-0,7947$ \\
\hline Primavera & 0,7142 & $-0,5526$ & $-0,7187$ \\
\hline
\end{tabular}

Tabela 5 - Resultados de coeficiente de correlação (escala anual).

\begin{tabular}{|c|c|c|c|}
\hline Ano & R (Observação x Estimativa) & $\mathrm{R}$ (Observação x Desvio 1) & R (Observação x Desvio 2) \\
\hline $1983-2008$ & 0,6814 & $-0,4300$ & $-0,6449$ \\
\hline
\end{tabular}

Para as observações nas escalas de tempo mensal, sazonal e anual, o indicador Desvio 2 apresentou melhores resultados. Ele representa uma normalização da diferença (entre observação e o estimado) pelo valor típico do mês (observado).

As Figuras 5 e 6 representam os melhores resultados obtidos em escala mensal e sazonal que foram, respectivamente, em maio $(\mathrm{R}=0,8890)$ e no período de inverno $(0,7725)$. Como pode ser notado, eles não apresentam distorções de resultados.

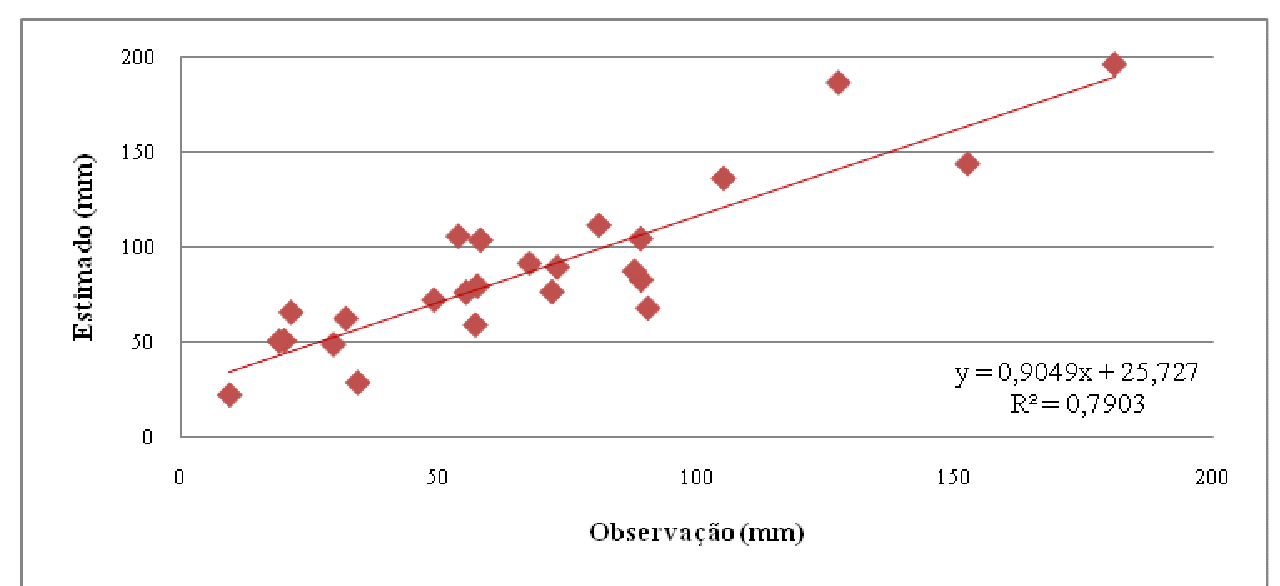

Figura 5 - Comparação entre dados observacionais (UNITAU) com a estimativa (GPCP) para o mês de maio. 


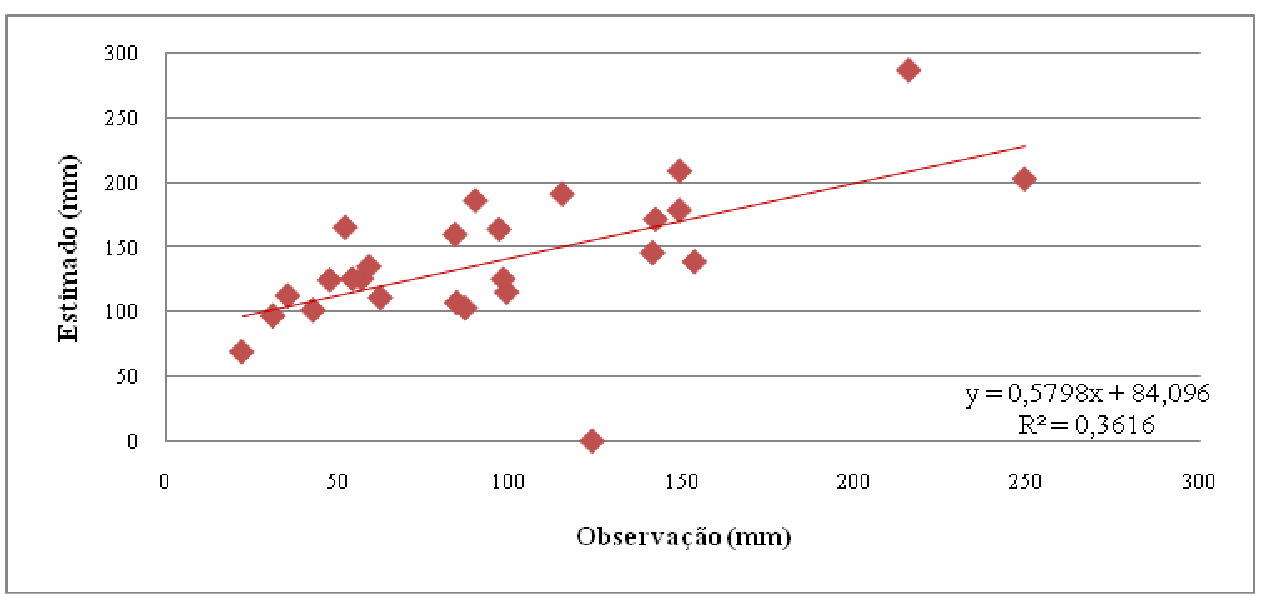

Figura 6 - Comparação entre dados observacionais (UNITAU) com a estimativa (GPCP) para o período de inverno.

\section{Conclusão}

Os resultados apresentados nas escalas de tempo mensal, sazonal e anual para a série temporal de observações e do GPCP mostram a mesma tendência, o que representa que os dados do GPCP podem ser utilizados como representativos da região do Vale do Paraíba.

A análise da série temporal completa do GPCP mostra que não houve alteração na tendência de precipitação, indicando a manutenção do regime pluviométrico local ao longo dos últimos 29 anos, sendo $\tau=-0,0394$.

Através das correlações mensais, sazonais e anuais foram obtidos os coeficientes de correlação e as retas de ajuste, bem como os desvios entre os dados, por meio dos quais se validou as estimativas do GPCP para valores observacionais do Vale do Paraíba. Isto permitiu a ampliação dos dados de precipitação para uma série histórica mais longa de 29 anos, suprindo as falhas e limitações destes. No período de 1983 a 2008, os melhores resultados correspondem ao mês de maio $(\mathrm{R}=0,8890)$ e ao inverno $(\mathrm{R}=0,7725)$. De modo geral, a correlação entre observações e estimativas ou desvios calculados para todas as escalas temporais analisadas são classificadas médias $(0,3 \leq R<0,7)$ ou altas $(0,7 \leq R<1)$.

As variações nos ajustes obtidos podem ser justificadas pela origem dos dados, lembrando que os dados estimados compreendem um quadrante de $2,5^{\circ}$ × $2,5^{\circ}$ e os dados observacionais são pontuais.

\section{Referências Bibliográficas}

Adler, R.F., G.J. Huffman, A. Chang, R. Ferraro, P. Xie, J. Janowiak, B. Rudolf, U. Schneider, S. Curtis, D. Bolvin, A. Gruber, J. Susskind, and P. Arkin, 2003: The Version 2 Global Precipitation Climatology Project (GPCP) Monthly Precipitation Analysis (1979 - Present). J. Hydrometeor, v. 4, n. 6, p. 1147-1167, 2003.

BRASIL. Ministério da Defesa. Centro de Lançamento de Alcântara. Disponível em: <http://www.cla.aer.mil.br/>. Acesso em: 16 jan. 2009.

Fisch, G. Vendrame, I. F., Hanaoka, P.C. de M. Variabilidade Espacial da Chuva durante o experimento LBA/TRMM 1999 na Amazônia. Acta Amazônica, v. 37, n. 4, p. 585-592, 2007. 
Folhes, M. T. Fisch, G. Caracterização Climática e Estudo de Tendências nas Séries Temporais de Temperatura do Ar em Taubaté (SP). Ambi Agua, 2006.

Gruber, A. e Levizzani, V. Assessment shows no trend in the global precipitation. Gewex News, v. 16, n. 4, p. 6-8, 2006.

National Aeronautics and Space Administration . Global Precipitation Climatology Project. Rainfall Analysis Tools. Disponível em: <http://disc2.nascom.nasa.gov/Giovanni/tovas/rain.GPCP.2.shtml>. Acesso em: 16 jan. 2009.

Vianello, R. L.; Alves, A. R. Meteorologia básica e aplicações. Viçosa: Imprensa Universitária, 1991. 